\title{
Erratic Multiplicity Fluctuation in Heavy-Ion Collisions at High Energy
}

\author{
Mohammed Mohisin Khan ${ }^{1, *}$, Danish Meer ${ }^{1}$, Tahir Hussain ${ }^{2}$, Arshad Kamal $^{3}$, Waseem Bari ${ }^{4}$ \\ ${ }^{1}$ Department of Applied Physics, Zakir Husain College of Engineering and Technology, Aligarh Muslim University, Aligarh, India \\ ${ }^{2}$ Applied Sciences and Humanities Section, University Polytechnic, Aligarh Muslim University, Aligarh, India \\ ${ }^{3}$ Department of Physics, Shibli National College, Azamgarh, India \\ ${ }^{4}$ Department of Physics, University of Kashmir, Srinagar, India
}

Email address:

mohsinkhan.ph@amu.ac.in(M. M. Khan)

${ }^{*}$ Corresponding author

\section{To cite this article:}

Mohammed Mohisin Khan, Danish Meer, Tahir Hussain, Arshad Kamal, Waseem Bari. Erratic Multiplicity Fluctuation in Heavy-Ion Collisions at High Energy. International Journal of High Energy Physics. Vol. 7, No. 2, 2020, pp. 37-40. doi: 10.11648/j.ijhep.20200702.12

Received: July 14, 2020; Accepted: August 6, 2020; Published: September 24, 2020

\begin{abstract}
The results reported in this manuscript are an attempt to look for the occurrence of erratic multiplicity fluctuations in pseudorapidity space of the secondary particles produced in ${ }^{16} \mathrm{O}$-nucleus interactions at an incident momentum of $3.7 \mathrm{~A} \mathrm{GeV} / \mathrm{c}$ and to compare our results with the finding of other researches on dynamical fluctuations at various projectile energies. The analysis heavily relies on the method of normalized factorial moments and the binning of the phase space. In this analysis we have obtained results on the normalized factorial moments and other physical parameters derived from them. Results obtained for the experimental data are compared with the results for FRITIOF simulated events. The results on $\mathrm{F}_{2}$ distribution, $\mathrm{C}_{\mathrm{pq}}$-moments and on $\Sigma_{\mathrm{q}}$ obtained in the present study hints towards the existence of chaoticity in the multiparticle production in high energy nucleus-nucleus collisions. Occurrence of such non-statistical fluctuations further suggests the presence of correlated particle production in relativistic nuclear collisions.
\end{abstract}

Keywords: Correlation and Fluctuation, Event-by-Event, High Energy Nuclear Collisions

\section{Introduction}

One of the main goals of the nuclear collisions at high energy is to produce and study the behaviour of the hot and dense nuclear matter. Various experiments in the past at worldwide and some current experiments such has STAR, PHOBOS and ALICE at the RHICH and LHC have produced a vast amount of data that rveal the production of quark-gluon plasma (QGP) which subsequently make transition to normal hadronic phase [1-7]. Event-by-event fluctuations of the global observable, in particular, are considered to be very useful tools to understand the QGPhadronic phase transition and to understand the underlying dynamics of the multiparticle production in general. Since fluctuations observables are intrinsically related to particle correlations, the study of fluctuations could also provide helpful insights on the mechanism of multiparticle production in relativistic nuclear collisions [1-3]. In the present study the erratic fluctuaion analysis has been performed using the power-law behaviour of the moments of the event factorial moment $\left(\mathrm{F}_{\mathrm{q}}\right.$-moment) distribution, for example, the normalized moment $\mathrm{C}_{\mathrm{p}, \mathrm{q}}$ which also exhibits a power-law behaviour with the decreasing pseudorapiidty bin. This behaviour is referred to as erraticity [8-10]. It may be stressed that erraticity analysis simultaneously takes into account spatial as well as e-by-e fluctuations beyond intermittency [8]. The erraticity analysis performed for the events simulated in the framework of perturbative QCD indicates that the behaviour of multiparticle production is chaotic [11]. Various analyses of experimental data on relativistic hadronic and nuclear collisions also reveals the occurrence of the erratic multiplicity fluctuations [9-13]. It may be stressed that these studies are still not conclusive as it is not clear whether these fluctuations have some dynamical origin or are purely statistical. It was, therefore, considered worthwhile to examine erraticity behaviour in 
relativistic AA collisions by examining experimental and simulated data on ${ }^{16} \mathrm{O}$-nucleus interactions at $3.7 \mathrm{~A} \mathrm{GeV} / \mathrm{c}$ incident momentum

\section{Methodology}

Cao and Hwa [11] have proposed to probe the phase space pattern of a produced multiparticle system by making the use of the factorial moments of the multiplicity distributions which can be defined as:

$$
F_{q}^{e}=\left[\frac{1}{M} \sum_{m=1}^{M} n_{m}\left(n_{m}-1\right) . .\left(n_{m}-q+1\right)\right] \cdot\left(\frac{1}{M} \sum_{m=1}^{M} n_{m}\right)^{-q}
$$

where $M$ is the number of partition chosen in the pseudorapidity space and $\mathrm{n}_{\mathrm{m}}$ denotes the number of particles in the $\mathrm{m}^{\text {th }}$ bin.

To quantify e-by-e fluctuations in terms of the event factorial moment, the normalised moments, $\mathrm{C}_{\mathrm{p}, \mathrm{q}}$ are calculated using

$$
C_{p q}=\left(\frac{F_{q}^{e}}{<F_{q>}^{e}}\right)^{p}
$$

The order of the moment $\mathrm{q}$, is an integer whereas $\mathrm{p}$ may take on any value greater than zero. If $\mathrm{C}_{\mathrm{pq}}$ exhibit power-law behaviour of the type

$$
C_{p q} \propto M^{\psi_{p(q)}}
$$

The power of $\mathrm{M}$ is known as the erraticity exponent and such a power law behavior of $\mathrm{C}_{\mathrm{p}, \mathrm{q}}$ is termed as the erraticity quantifying the presence of erratic fluctuations. Another important parameter is an entropy like quantity, $\sum_{q}$ which define the degree of erraticity or chaoticity and can be expressed as

$$
\Sigma_{q}=\Phi_{q} \ln \Phi_{q}
$$

where $\Phi_{q}=\frac{<F_{q>}^{e}}{F_{q}^{e}}$, the positive values of $\sum_{\mathrm{q}}$ are regarded as the criteria for measuring the chaos [11].

\section{Details of the Data}

The experimental data used in the present study is the one obtained from emulsions experiments. A random sample comprising of 297 events having $n_{s}>2$, where $n_{s}$ represents the number of relativistic charged particles in an event, produced in $3.7 \mathrm{~A} \mathrm{GeV/c}{ }^{16} \mathrm{O}$-nucleus interactions, is used for carrying out the present analysis. The emission angles of all the relativistic charged particles were measured and their pseudorapidities were determined. All other necessary details about the stacks used, criteria for selecting the events and the method of measuring the emission angles may be found elsewhere [15]. Furthermore, comparing the experimental results with the corresponding values predicted by the Lund model, a sample consisting of 3000 events, identical to the experimental ones were simulated using the FRITIOF code [16].

\section{Results and Discussion}

In order to examine event-by-event fluctuations the values of $F_{q}^{e}$ are calculated for various values of $\mathrm{M}$ for the Experimental and FRITIOF data and their distributions for $\mathrm{M}=5,10$ and 20 are shown in Figure 1. It is observed from the figure 1 that the $F_{2}$ distributions have a noticebale peak at around $\mathrm{F}_{2} \approx 1.2$ and tends to become broader with increasing $M$. This might imply that with increasing $M$ or decreasing pseudorapiidty bin size e-bye-e fluctuations tend to become relatively broader. It is also noticebale from the figure that these fluctuations for the experimental data are comparatively broader in comparison to those for the FRITIOF events for the three values of $M$ being. Similar trends of $F_{q}^{e}$ distributions have also been reported for the $\mathrm{MC}$ simulation of jet fragments of the perturbative QCD [11].

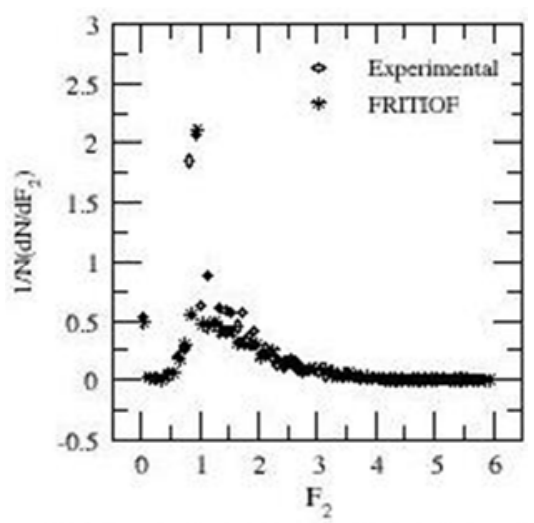

Figure 1. $F_{2}$-distribution for the experimental and simulated data

To proceed further for examining the erratic nature of the fluctuation, the values of $\mathrm{C}_{\mathrm{pq}}$-moments have been determined for $\mathrm{q}=2,3$ and 4 . The variation of the natural log of these moments, $\ln \mathrm{C}_{\mathrm{p}, \mathrm{q}}$, with $\ln \mathrm{M}(\mathrm{M}=1,10)$ for both experimental and FRITIOF data is depicted in Figure 2. The dependence of $\mathrm{C}_{\mathrm{p}, \mathrm{q}}$ on $\mathrm{p}$ is considered to provide interesting information on the erratic fluctuations and for studying the same the values of $\mathrm{C}_{\mathrm{p}, \mathrm{q}}$ are calculated for $\mathrm{p}$ lying in the interval $0.5-2.1$ in a step of 0.2 , but in the figure the values of $C_{p, q}$ for $p=0.5,0.9$, 1.3 and 2.1 only are plotted to avoid over crowding of lines and symbols. Statistical errors are also shown in Figure 2.

It is evident from Figure 2 that the shapes of $\ln C_{p, q}$ vs $\ln M$ plots are essentially same for both the experimental and FRITIOF data, therby showing the presence of erraticity in the multiparticle production in ${ }^{16} \mathrm{O}$-nucleus interactions at 3.7A GeV/c. Another useful parameter determining the erraticity is $\sum_{q}$. The values of $\sum_{q}$ are determined according to the formula given in the methodology section and the dependence of this variable on $\operatorname{lnM}$ for $\mathrm{q}=2,3$ and 4 is displayed in Figure 3.

Again the dependence of $\sum_{2}, \sum_{3}$ and $\sum_{4}$ on $\ln \mathrm{M}$ is almost same for the experimental and FRITIOF simulated data. The trends of variations of $\mathrm{C}_{\mathrm{p}, \mathrm{q}}$ with $\mathrm{M}$ and $\mathrm{p}$ and the trends of variation $\sum_{q}$ with $\ln M$ supports the presence of event-by-event multipliicty fluctuations in rapiidty space and that the 
fluctuations are eeratic in nature. Similar observations have also be made by other workers at different energies in $\mathrm{GeV}$ range and
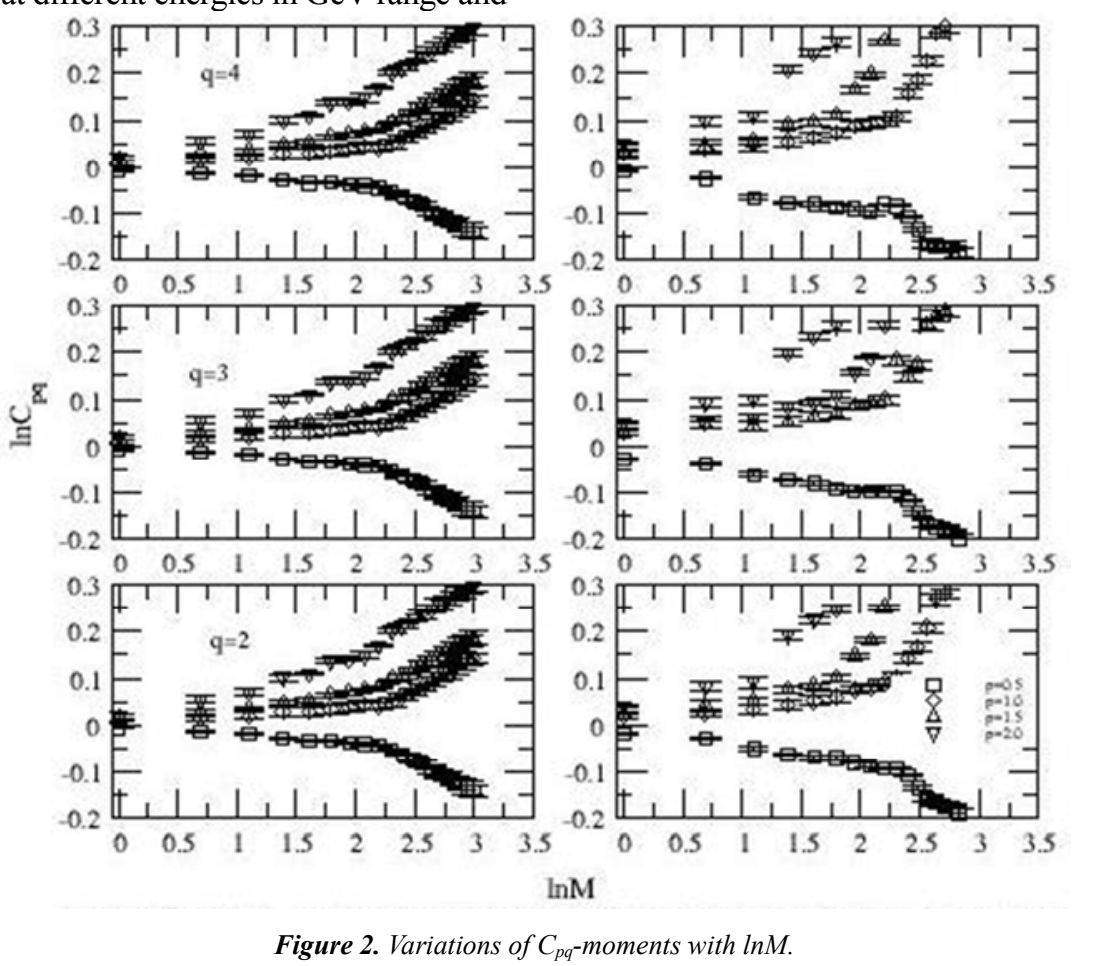

Figure 2. Variations of $C_{p q}$-moments with $\ln M$.
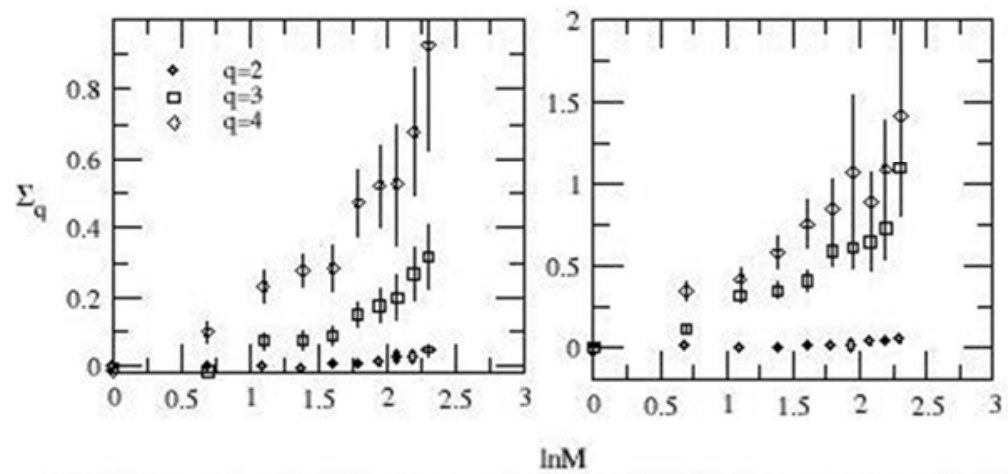

Figure 3. Variation of $\sum_{q}$ with $\ln M$ for $q=2,3$ and 4 .
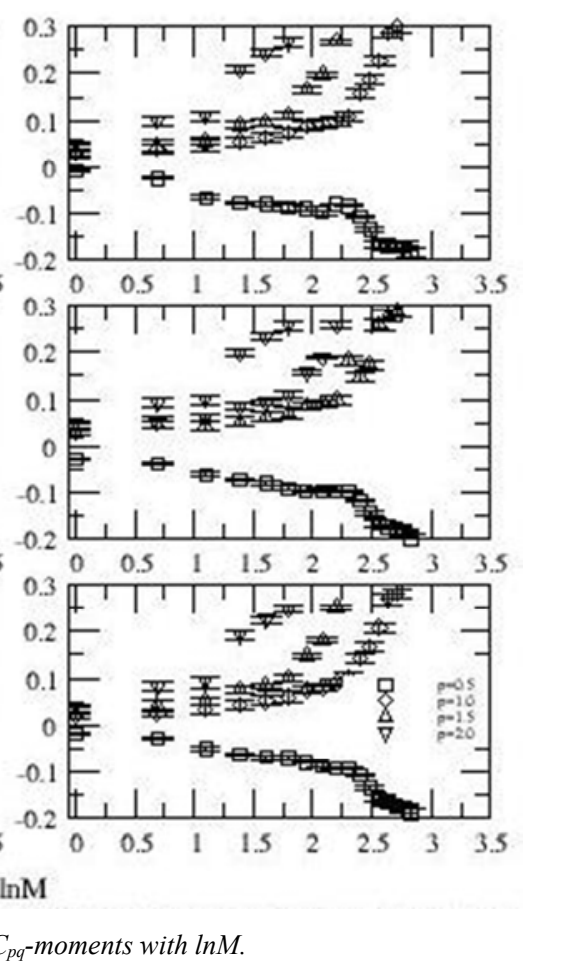

with different colliding systems. [10, 11, 14, 15, 17-21].$$
\text { . }
$$

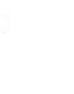


[7] R. Halynski et. al. (KLM Collaboration), Phys. Rev. Lett. 62, (1989) 733: M. I. Adamovich et. al. (EMU01 Collaboration), Phys. Rev. Lett. 263, (1991) 539; Nucl. Phys. B388, (1992) 3.

[8] E. A. De. Wolf, I. M. Dremin and W. Kittle, Phys. Rep. \{lbf $270\},(1996) 48$.

[9] R. C. Hwa, Acta Phy. Pol. B27, (1996) 1789.

[10] L. Fuming, L. Hongbo, L. Ming, L. Ferig and L. Lianshou Phys. Lett B576, (2001) 293.

[11] F. Jinghua, W. Yuanfang and L. Lianshou Phys. Lett B472, (2000) 161.

[12] Z. Cao and R. C. Hwa Phys. Rev. Lett. 75, (1995) 1268: Phys. Rev. D53, (1996) 6608.

[13] M. R. Atayan et al (EHS/NA22 Collaboration) Phys. Lett. B558, (2003) 22 .
[14] M. K. Ghosh and A. Mukhopadhayay, Phys. Rev. C68, (2003) 034907.

[15] Shakeel Ahmad, M. M. Khan, N. Ahmad, M. Zafar and M. Irfan, Acta. Phys. Pol. 35, (2004) 809.

[16] B. Nilsson-Almqvist, E. Stenlund, Comput. Phys. Commun. 43, 387 (1987).

[17] D. Ghosh, A. Deb, M. Mondal and J. Ghosh Phys. Lett B540, (2002) 52; Phys Rev. C68, (2003) 0249089; J. Phys. G: Nucl. Part. Phys. 29, (2003) 2087.

[18] Shaoshun W. and Zhaomin W., Phys. Rev. D57, (1998) 3036.

[19] Proc. $7^{\text {th }}$ Int. Conf. on Multiparticle Production-Correlations and Fluctuations, Ni-jimegen, June 30-July 06, 1996. p. 321.

[20] Z. Cao and R. C. Hwa, Phys. Rev. D54, (1996) 6674.

[21] Zhou Yi-Fei et al 2001 Chin. Phys. Lett. 18 (2001) 1179. 